\title{
Charles-Joseph Prince de Ligne, Mon Journal de la guerre de Sept Ans
}

\section{Maria Immacolata Spagna}

\section{(2) OpenEdition}

1 Journals

\section{Edizione digitale}

URL: http://journals.openedition.org/studifrancesi/7589

DOI: $10.4000 /$ studifrancesi.7589

ISSN: 2421-5856

\section{Editore}

Rosenberg \& Sellier

\section{Edizione cartacea}

Data di pubblicazione: 1 décembre 2009

Paginazione: 631-632

ISSN: 0039-2944

\section{Notizia bibliografica digitale}

Maria Immacolata Spagna, «Charles-Joseph Prince de Ligne, Mon Journal de la guerre de Sept Ans»,

Studi Francesi [Online], 159 (LIII | III) | 2009, online dal 30 novembre 2015, consultato il 08 janvier 2021. URL: http://journals.openedition.org/studifrancesi/7589; DOI: https://doi.org/10.4000/studifrancesi. 7589

Questo documento è stato generato automaticamente il 8 janvier 2021.

\section{(c) $(1) \ominus$}

Studi Francesi è distribuita con Licenza Creative Commons Attribuzione - Non commerciale - Non opere derivate 4.0 Internazionale. 


\title{
Charles-Joseph Prince de Ligne, Mon Journal de la guerre de Sept Ans
}

\author{
Maria Immacolata Spagna
}

\section{NOTIZIA}

CHARLES-JOSEPH PRINCE DE LIGNE, Mon Journal de la guerre de Sept Ans. Textes inédits introduits, établis et annotés par Jeroom VERCRUYSSE et Bruno COLSON, Paris, Champion, 2008 («L’Âge des Lumières», 44), pp. 552.

1 Mon Journal de la guerre de Sept Ans è il titolo che il principe Charles-Joseph de Ligne (1735-1814) ha dato alla sua relazione sulla guerra dei Sette Anni (1756-1763) di cui fu un attore e un testimone attivo. Il principe, allora giovane ufficiale al servizio dell'Austria, prese parte alle campagne nell'Europa centrale e fu coinvolto in numerosi combattimenti. Da questa esperienza, prima come capitano, più tardi in veste di luogotenente-colonnello e infine in quanto colonnello comandante dei «Wallons», il Prince de Ligne ha tratto la materia per un giornale nel quale ha raccontato gli avvenimenti vissuti, espresso le sue osservazioni strategiche, distribuito elogi e critiche nei confronti dei suoi soldati, superiori o capi e dell'avversario. Prendendo le distanze dagli storiografi quali Boileau, Racine, Voltaire, il principe non intendeva scrivere la storia della guerra dei Sette Anni ma riportare ciò che aveva realmente visto e vissuto.

Il giornale ha conosciuto un'unica edizione, curata dallo stesso de Ligne nel 1796, che occupa i tomi XIV, XV e XVI dei suoi Mélanges militaires, littéraires et sentimentaires. Tale pubblicazione, realizzata una trentina di anni dopo la fine della guerra, offre una versione largamente rivista di una prima redazione senza titolo, manoscritta, anonima e inedita fino ad oggi, conservata negli archivi del Service historique de la Défense, Département de l'Armée de Terre, a Vincennes.

La presente edizione critica propone, per la prima volta, in un volume a parte, le due versioni, quella manoscritta e quella stampata. Il confronto tra le due permette di 
coglierne le differenze e di notare come la versione successiva sia meno dura e franca rispetto a quella manoscritta.

4 La versione stampata è molto più lunga rispetto a quella manoscritta, il cui racconto si ferma verso la metà di luglio: le battaglie di Liegnitz (agosto) e Torgau (novembre) non sono evocate. La versione manoscritta è stata realizzata da più copisti e presenta un'ortografia piuttosto approssimativa, quasi fonetica, soprattutto quando si tratta di nomi di militari o di qualche luogo. Quella della versione stampata è invece più uniforme, anche se l'ortografia dei nomi e delle parole straniere resta esitante.

Per quanto riguarda il contenuto, una seria rilettura del manoscritto del Journal si impone in vista della sua pubblicazione, anche perché il teatro dell'Europa nel frattempo era cambiato. Molti dettagli poco favorevoli all'armata austriaca sono spariti. Con il passare del tempo, gli incontri di de Ligne con gli antichi avversari dell'Austria, Federico II, suo fratello Henri e altri generali, gli hanno permesso di discutere e confrontarsi sugli avvenimenti del passato. Egli è cosciente che alcune sue affermazioni rischiano di dispiacere. Mentre nella versione manoscritta non risente di tali scrupoli e non esita a nominare i responsabili di errori e di fallimenti, di denunciare mancanza di preparazione, di coordinazione, di decisione, e anche di ufficiali capaci, in quella stampata modifica un po' il suo modo di giudicare.

6 C'è da dire comunque che anche se una nuova edizione è evidente nel 1796, essa non stravolge totalmente l'opera intrapresa anni prima, quando niente lasciava presagire gli sconvolgimenti che la società avrebbe conosciuto qualche decennio più tardi. L'autoritratto, che viene fuori dal racconto si conferma nelle due versioni: quello di un uomo coraggioso, temerario, portato all'azione, in collera quando non riesce a convincere i superiori, di cattivo umore se non può prendere parte ad un'azione importante, che prova soddisfazione dopo le vittorie conseguite come quella di Kolin del 18 giugno 1757 o di Hochkirch del 14 ottobre 1758, che si abbatte per una disfatta come quella di Leuthen il 5 dicembre 1757, che prova pietà per i villaggi di Zittau o di Zinna distrutti dalle fiamme, che si commuove di fronte ai cadaveri delle vittime civili.

7 L'impressione che dà dell'armata austriaca è sempre quella di un esercito pesante e soprattutto poco intraprendente, che si accontenta di scegliere una buona posizione e di difenderla. La Prussia guidata da Federico II appare, invece, audace e fa paura all'avversario. Non è un caso che il racconto di de Ligne termini proprio con un elogio al re di Prussia.

In appendice, si trova un documento che costituisce un prezioso supplemento al Journal, le Réflexions inédites sur la guerre de Sept Ans (1812), un testo inedito delle riflessioni tardive del principe sulle campagne della guerra dei Sette Anni, conservato negli archivi della Maison de Ligne. Scritte quasi mezzo secolo dopo gli avvenimenti, queste note critiche sono il risultato delle considerazioni alle quali il principe giunge dopo la lettura di opere relative alla guerra, in particolare quella del generale maggiore britannico Henry Humphrey Evans Lloyd, The History of the late War in Germany, between the King of Prussia and the Empress of Germany and her Allies (1766).

Nelle Annexes si trovano le carte geografiche di alcune battaglie della guerra ed il quadro delle colonne d'attacco austriache alla battaglia di Hochkirch, così come le dà il principe de Ligne nella versione stampata del suo Journal. Alla fine del volume, un indice completo e dettagliato che tenta di ristabilire le grafie esatte di tutti i nomi delle persone e dei luoghi citati nell'opera. 
10 Mon Journal de la guerre de Sept Ans costituisce la prima opera storica del principe de Ligne, una personalità poco comune che continua ad essere oggetto di studio per molti cultori e ricercatori. 